\title{
Functional dentition and changes in dietary patterns among older adults in Thailand
}

\author{
Piyada Gaewkhiew ${ }^{1,2, *}$, Wael Sabbah ${ }^{1}$ and Eduardo Bernabé ${ }^{1}$ \\ 'Faculty of Dentistry, Oral \& Craniofacial Sciences, King's College London Dental Institute at Guy's, King's College and \\ St. Thomas' Hospitals, London SE5 9RS, UK: '2Faculty of Dentistry, Department of Community Dentistry, Mahidol \\ University, Ratchathewi, 10400 Bangkok, Thailand
}

Submitted 5 June 2020: Final revision received 8 0ctober 2020: Accepted 20 0ctober 2020: First published online 27 October 2020

\begin{abstract}
Objective: To investigate the relationship between functional dentition (FD) and changes in dietary patterns (DP) in older adults.

Design: This was a 12-month prospective study, with dental examinations at baseline and questionnaires at baseline and follow-up. Dentition was classified as FD (containing $\geq 10$ occlusal contacts), non-FD with dentures and non-FD without dentures. A 154-item FFQ assessed dietary intake in the previous month. Food items (servings/d) were combined into twenty-two food groups based on their similar nutrient profile, culinary use and previous studies in Thailand. DP were identified through factor analysis of baseline intake and applied scores were used to estimate changes in DP scores. The association between baseline FD (exposure) and change in each DP score (outcome) was tested in linear regression models adjusting for baseline socio-demographic factors, behaviours, chronic conditions, medications, total energy intake and DP score.

Setting: Phetchaburi, Thailand.

Participants: Totally, 788 community dwellers aged $\geq 60$ years.

Results: In total, 651 participants were retained after 12 months (82.6\% retention rate), of whom $14.1 \%$ had FD. Having an FD was positively associated with larger increases in vegetable intake. Three DP were identified. Participants with FD had larger increases in healthy $(0 \cdot 13 ; 95 \% \mathrm{CI}:-0 \cdot 13,0 \cdot 39)$ and carbohydrate-rich diets intake $(0 \cdot 12 ; 95 \% \mathrm{CI}:-0 \cdot 17,0 \cdot 40)$ as well as larger reductions in meat-rich diet intake $(-0.12 ; 95 \% \mathrm{CI}:-0.45,0.21)$ than those with neither FD nor dentures. However, these differences were not significant.

Conclusion: There was little support for an association between baseline FD and changes in DP.
\end{abstract}

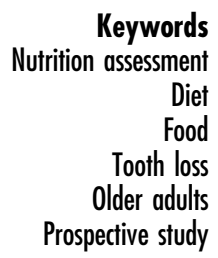

Healthy ageing is the process of developing and maintaining the functional ability that enables well-being in older age, which can be achieved by preventing chronic disease and maintaining physical and mental functions throughout life ${ }^{(1)}$. Given that common chronic conditions, such as obesity, CVD, diabetes and cancer, are related to nutritional factors, having a balanced and healthy diet that provides enough energy to meet daily demands is vital to achieve these goals ${ }^{(2)}$.

Oral health is an essential component of general health and plays an important role in healthy ageing ${ }^{(3)}$. However, three recent systematic reviews provided inconclusive evidence for the association of mastication ${ }^{(4)}$, tooth loss ${ }^{(5)}$ and oral function ${ }^{(6)}$ with dietary and nutrient intake among independently living older adults. Most studies identified were cross sectional and used the count of teeth (either self-reported or clinically determined) which does not accurately reflect the distribution of teeth within the mouth and their functioning ${ }^{(7)}$. A functional dentition (FD), i.e. one that consists of all six anterior occlusal contacts and at least four (out of a maximum of sixteen) posterior occlusal contacts, allows individuals to satisfy masticatory, appearance, comfort and social requirements ${ }^{(8)}$. The only previous longitudinal study measuring FD found greater reductions in intake of nutrients (protein, fibre, vitamins A and E, Ca, $\mathrm{Na}$ and $\mathrm{K}$ ) and food groups (vegetables and meat) after 5 years among Japanese older adults without FD than among those with $\mathrm{FD}^{(9)}$. 
A possible explanation for these inconclusive findings has to do with the emphasis on the role of single nutrients (protein or fibre) or foods (fruits and vegetables) over the role of the entire diet of individuals ${ }^{(10,11)}$. A whole diet assessment can better explain habitual food consumption because humans consumed foods as a combination or a variety of foods ${ }^{(11)}$. The analysis of DP recognises the complexity of diets, potential additive effects between different food groups, as well as the variability in DP existing within and between populations ${ }^{(10)}$. DP can be derived either a priori or a posterior $i^{(12)}$. The a priori approach evaluates the healthiness of the diet based on current nutrition guidelines, which are then aggregated to produce a summary measure of dietary quality of individuals ${ }^{(13)}$. The a posteriori approach is based on multivariate data reduction techniques that reveal underlying common food consumption patterns ${ }^{(14)}$. There is growing evidence that a diverse diet may help prevent chronic conditions ${ }^{(15,16)}$. However, to the best of our knowledge, no previous dental study has explored whether FD can play a role in explaining DP. To fill this gap in knowledge, a study was conducted to investigate the association between FD and changes in DP among Thai older adults.

\section{Methods}

This report is based on data from a 12-month prospective study conducted among older adults in Phetchaburi province, Thailand. All participants signed a written informed consent.

\section{Study population}

In 2018, health authorities in four of the eight districts in Phetchaburi agreed to participate in the study. In these four districts, six urban and eleven rural sub-districts (out of sixteen and thirty nine, respectively) agreed to participate. Overall, 788 Thai adults, aged 60 years and over, were recruited ( $97 \%$ response rate) via social media websites and community meetings. Participants were included if they were Thai nationals, registered with the healthcare system and had scores $\geq 8$ in the Abbreviated Mental Test Score ${ }^{(17)}$ and $\geq 12$ in the Activities of Daily Living index ${ }^{(18)}$. Both instruments are routinely used in primary care in Thailand. Participants were excluded if they could not communicate or had disabling conditions. All participants were invited to a follow-up survey a year later, of which 651 participants were re-assessed ( $83 \%$ retention rate). The mean follow-up time was 11.6 months (SD: 0.8, range: 10-13).

A minimum sample of 298 participants (60 with and 238 without FD) was required to estimate a moderate size effect (standardised mean difference of 0.50 units between groups), assuming the outcome was measured over two time points, the correlation between outcome measurements was high $(r=0.90), 95 \% \mathrm{CI}$ and $80 \%$ statistical power ${ }^{(19)}$. Sample size was increased to 373 (75 exposed and 298 unexposed) to compensate for a possible attrition rate of $25 \%$.

\section{Data collection}

Data were collected in primary healthcare centres via dental examinations at baseline and questionnaires at baseline and follow-up. The questionnaire collected information on demographic characteristics (sex, age and residence area), socio-economic circumstances (education and wealth index), behaviours (smoking status and physical activity) and chronic conditions. Ownership of ten household assets (air conditioner, bed, car, boiler, kettle, flushing toilet, house telephone, microwave, personal computer and washing machine) was assessed to derive the wealth index. Principal component analysis was used to extract the first common factor, which was subsequently used to obtain the wealth score and recoded it into quartiles ${ }^{(20)}$. Smoking status was determined with three questions on current and past cigarette consumption, from which participants were classified as never, former and current smokers ${ }^{(21)}$. Physical activity was determined with four questions on weekly frequency of strenuous, moderate, mild and walking exercise. Responses were used to obtain a weighted measure of overall metabolically adjusted exercise-related physical activity, calculated as $2 \times$ strenuous + moderate + mild + walking exercise sessions ${ }^{(22)}$. Participants' physical activity was classified as $0-6,7$ and $8+$ sessions/week. Participants also reported whether they have ever been diagnosed with hypertension, hyperlipidaemia and diabetes, which are the three most common chronic conditions among older adults in Thailand ${ }^{(23)}$. If a condition was reported, information on any medications taken was also collected from participants.

The questionnaire also included a 154-item semiquantitative FFQ to assess intake of food and beverages in the last month. The FFQ was specifically devised for use among Thai older adults ${ }^{(24)}$. The Thai elderly-FFQ was validated by comparing total energy intake and intake of protein, fat, $\mathrm{Ca}$ and $\mathrm{Fe}$ against $3 \mathrm{~d}$ of 24 -h dietary recalls. The Spearman's correlation coefficients between the two methods were 0.83 for energy, 0.69 for protein, 0.42 for fat, 0.47 for $\mathrm{Ca}$ and 0.28 for $\mathrm{Fe}^{(25,26)}$. It covered fifteen main dietary groups: (1) rice/carbohydrate-based dishes and ready meals; (2) beans and tofu products; (3) fresh and processed vegetables; (4) fresh and processed fruits; (5) meat and meat products; (6) fish and seafood; (7) egg and egg products; (8) insects; (9) milk and dairy products; (10) beverages and drinks; (11) sweet snacks and desserts; (12) snacks containing legumes, beans and seeds; (13) sauces and seasonings; (14) chilli paste and other pastes and (15) Thai curry and soups. For each food item, participants reported their frequency of consumption using seven-point ordinal scales (from never to three or more times a d) and portion size using seven-point ordinal 
scales (from a quarter of a portion to two standard portions). They were assisted to estimate portion sizes using pictures of portion sizes and cooking measurements. The fifteen FFQ groups were further divided to create twenty-two food groups based on their similar nutrient profile, culinary use and previous studies in Thailand ${ }^{(27,28)}$. All food groups with their constituent food items are shown in the Appendix. Daily servings were estimated by multiplying portion size and frequency of consumption of food items. These were then summed across all items within a group to reflect participants' habitual consumption of that food group. Intake of food groups (servings/d) was estimated at baseline and follow-up separately.

Dental examinations were conducted to determine the number of functional tooth units (FTU), the condition of teeth and dentures. Two trained dentists examined participants on mobile dental chairs, under artificial light and using a mouth mirror and periodontal probe. An FTU was recorded as present if there were opposing teeth (natural or replaced with fixed prostheses). Opposing molars (including wisdom teeth) counted as two FTU, whereas premolars, canines and incisors counted as one FTU each. Dental caries was diagnosed according to the WHO criteria ${ }^{(29)}$. In addition, teeth with severe mobility (i.e. $>2 \mathrm{~mm}$ of buccolingual or any vertical movement ${ }^{(30)}$ ) were recorded. Removable complete and partial dentures were recorded as present if they were worn on the day of the examination and reported to be worn while eating food. FD was defined as having all six anterior FTU plus at least four (out of a maximum of sixteen) posterior FTU. An FTU was not counted as part of the ten or more FTU needed if it had a tooth with severe caries (root remnants) or severe mobility ${ }^{(20,31)}$. Participants' dentition was classified as functional, non-functional with dentures and non-functional without dentures (reference group for comparisons).

\section{Statistical analysis}

All analyses were conducted in Stata 16 (Stata Corp.). We first compared participants who were successfully retained after 12 months with those lost to follow-up, using $\chi^{2}$ test. We then compared the socio-demographic characteristics, behaviours, chronic conditions and medications taken between the three FD groups, using the $\chi^{2}$ test. Thereafter, the association between baseline FD (exposure) and changes in intake of food groups (outcome) was evaluated in adjusted linear regression models. The adjusted models included controls for baseline sociodemographic characteristics (age groups, gender, residence area, education and wealth quartiles), behaviours (smoking status and physical activity), chronic conditions (hypertension, hyperlipidaemia and diabetes), medications taken, total energy intake and food group intake.

We used applied scores to estimate changes in DP over time ${ }^{(32,33)}$. This approach is useful in longitudinal studies where the number of factors extracted and factor loadings would certainly change ${ }^{(32)}$. Indeed, we identified different DP at baseline ad follow-up in terms of factors extracted, food groups describing them and size of factor loadings. Three steps were followed. First, exploratory factor analysis of the baseline intake of twenty-two food groups was conducted to identify food groups that loaded highly (factor loadings $>0.25$ ) on a factor. The number of factors was determined based on an eigenvalue $>1$, the scree plot and interpretability ${ }^{(10,11)}$. Factors were rotated with an orthogonal (varimax) rotation to minimise correlation between factors and improve interpretability ${ }^{(11,14)}$. Second, standardised factor scores for DP were computed by adding intakes of food groups weighted by their factor loadings. Third, applied dietary pattern scores at follow-up were calculated by multiplying each participant's intake of food groups at follow-up, which was standardised to the mean and SD of baseline intakes, by the coefficients from the factor analysis of baseline data, and summing across all food groups. With applied scores, the changes in factor scores reflect actual differences in the intake of food groups within the factor (dietary pattern) rather than a change in the participants' position relative to the group average intake ${ }^{(34)}$.

The crude and adjusted association between baseline FD (exposure) and changes in dietary pattern scores (outcome) was tested in linear regression models. The adjusted models included controls for baseline sociodemographic factors, behaviours, chronic conditions, medications, total energy intake and dietary pattern score.

\section{Results}

Six hundred and fifty one of the 788 participants at baseline were retained after 12 months. Participants lost to followup were significantly more likely to be men, from rural areas, smokers and non-diabetic as well as less likely to take multiple medications than those retained in the sample. In all, $14 \cdot 1 \%$ of participants had FD, $20 \cdot 1 \%$ had non-FD with dentures and $65.7 \%$ had non-FD without dentures. The comparison of baseline characteristics by FD groups is shown in Table 1. Participants who had non-FD without dentures were older and took more medications than those in the other two groups. No other differences between the three FD groups were noted.

Table 2 shows average changes in daily intakes for twenty-two food groups by FD groups. Of the twentytwo groups assessed, FD was associated with intake of vegetables only. Participants with FD at baseline had larger increases in intake of vegetables than those who had non-FD without dentures ( $1.33 v \cdot 1.14$ servings/d). This association remained significant after adjustments for confounders (adjusted regression coefficient: 0.16; $95 \%$ CI: 0.04-0.28).

Three DP were identified through factor analysis of the intake data at baseline (Table 3), which explained $24.2 \%$ of 
Table 1 Comparison of socio-demographic characteristics, behaviours and chronic conditions by functional dentition (FD) groups ( $n$ 651)

\begin{tabular}{|c|c|c|c|c|c|c|c|}
\hline & \multicolumn{2}{|c|}{ FD-/D - $(n 428)$} & \multicolumn{2}{|c|}{ FD-/D + $(n$ 131) } & \multicolumn{2}{|c|}{$\mathrm{FD}+(n 92)$} & \multirow[b]{2}{*}{$P$ value } \\
\hline & $n$ & $\%$ & $n$ & $\%$ & $n$ & $\%$ & \\
\hline Gender & & & & & & & 0.212 \\
\hline Male & 125 & $19 \cdot 2$ & 28 & $4 \cdot 3$ & 25 & 3.8 & \\
\hline Female & 303 & 46.5 & 103 & $15 \cdot 8$ & 67 & $10 \cdot 3$ & \\
\hline Age & & & & & & & 0.006 \\
\hline $60-64$ years & 128 & $19 \cdot 7$ & 33 & $5 \cdot 1$ & 40 & $6 \cdot 1$ & \\
\hline $65-69$ years & 96 & 14.7 & 24 & 3.7 & 27 & $4 \cdot 1$ & \\
\hline 70-74 years & 78 & $12 \cdot 0$ & 33 & $5 \cdot 1$ & 12 & $1 \cdot 8$ & \\
\hline $75-79$ years & 65 & $10 \cdot 0$ & 19 & $2 \cdot 9$ & 8 & $1 \cdot 2$ & \\
\hline $80+$ years & 61 & $9 \cdot 4$ & 22 & 3.4 & 5 & 0.8 & \\
\hline Residence area & & & & & & & 0.144 \\
\hline Municipal & 200 & $30 \cdot 7$ & 71 & 10.9 & 38 & $5 \cdot 8$ & \\
\hline Non-municipal & 228 & $35 \cdot 0$ & 60 & $9 \cdot 2$ & 54 & $8 \cdot 3$ & \\
\hline Education & & & & & & & 0.460 \\
\hline No education & 54 & $8 \cdot 3$ & 14 & $2 \cdot 2$ & 15 & $2 \cdot 3$ & \\
\hline Any education & 374 & 57.5 & 117 & $18 \cdot 0$ & 77 & 11.8 & \\
\hline Wealth quartiles & & & & & & & 0.438 \\
\hline Q1 (poorest) & 110 & $16 \cdot 9$ & 26 & $4 \cdot 0$ & 27 & $4 \cdot 1$ & \\
\hline Q2 & 114 & 17.5 & 29 & 4.5 & 23 & 3.5 & \\
\hline Q3 & 104 & $16 \cdot 0$ & 37 & 5.7 & 23 & 3.5 & \\
\hline Q4 (wealthiest) & 100 & $15 \cdot 4$ & 39 & $6 \cdot 0$ & 19 & 2.9 & \\
\hline Physical activity & & & & & & & 0.380 \\
\hline 0-6 sessions/week & 49 & 7.5 & 14 & $2 \cdot 2$ & 12 & $1 \cdot 8$ & \\
\hline 7 sessions/week & 269 & $41 \cdot 3$ & 78 & $12 \cdot 0$ & 48 & $7 \cdot 4$ & \\
\hline $8+$ sessions/week & 110 & $16 \cdot 9$ & 39 & $6 \cdot 0$ & 32 & 4.9 & \\
\hline Smoking status & & & & & & & 0.183 \\
\hline Never & 305 & 46.9 & 104 & $16 \cdot 0$ & 64 & 9.8 & \\
\hline Former & 82 & $12 \cdot 6$ & 21 & 3.2 & 22 & 3.4 & \\
\hline Current & 41 & $6 \cdot 3$ & 6 & 0.9 & 6 & 0.9 & \\
\hline Hypertension & & & & & & & 0.062 \\
\hline No & 71 & $10 \cdot 9$ & 31 & $4 \cdot 8$ & 23 & 3.5 & \\
\hline Yes & 357 & $54 \cdot 8$ & 100 & $15 \cdot 4$ & 69 & $10 \cdot 6$ & \\
\hline Hyperlipidaemia & & & & & & & 0.477 \\
\hline No & 160 & $24 \cdot 6$ & 56 & 8.6 & 33 & $5 \cdot 1$ & \\
\hline Yes & 268 & $41 \cdot 2$ & 75 & 11.5 & 59 & $9 \cdot 1$ & \\
\hline Diabetes & & & & & & & 0.436 \\
\hline No & 272 & $41 \cdot 8$ & 91 & $14 \cdot 0$ & 58 & 8.9 & \\
\hline Yes & 156 & $24 \cdot 0$ & 40 & $6 \cdot 1$ & 34 & $5 \cdot 2$ & \\
\hline Medications & & & & & & & 0.005 \\
\hline None taken & 49 & 11.4 & 16 & $12 \cdot 2$ & 16 & $17 \cdot 4$ & \\
\hline $1-2$ taken & 181 & $42 \cdot 3$ & 74 & $56 \cdot 5$ & 29 & 31.5 & \\
\hline 3-4 taken & 157 & $36 \cdot 7$ & 34 & $26 \cdot 0$ & 41 & 44.6 & \\
\hline $5+$ taken & 41 & 9.6 & 7 & $5 \cdot 3$ & 6 & 6.5 & \\
\hline
\end{tabular}

FD-/D-, non-FD without dentures; FD-/D+, non-FD with dentures.

${ }^{*} \chi^{2}$ test was used for comparisons.

the variance in the twenty-two food groups. Pattern 1 was consistent with a healthy diet (i.e. it included high factor loadings for vegetables, fish and fermented fish/soybean, fruits, poultry, animal organs and fatty meat), pattern 2 was consistent with a diet rich in meat (positive loadings for red meat, poultry, processed meat, fatty meat, eggs, milk, shellfish and squid, food with coconut milk and negative loading for soymilk) and pattern 3 was consistent with a diet rich in carbohydrates (positive loadings for noodles, food with coconut milk, fruits, fried food and sweet snacks and negative loading for rice).

The factor loadings in Table 3 were used to estimate changes in DP using applied scores. Overall, there were increases in intakes of the healthy $(0.21 ; 95 \% \mathrm{CI}: 0.09$, $0.31)$ and carbohydrate-rich diets (0.60; $95 \%$ CI: 0.48 , 0.71 ) but no changes in intake of the meat-rich diet
$(-0 \cdot 11 ; 95 \% \mathrm{CI}:-0 \cdot 24,0 \cdot 01)$. Table 4 shows the association between baseline FD and changes in dietary pattern scores. Participants with FD had larger increases in intake of the healthy $(0 \cdot 13 ; 95 \% \mathrm{CI}:-0 \cdot 13,0 \cdot 39)$ and carbohydrate-rich diets $(0 \cdot 12 ; 95 \% \mathrm{CI}:-0 \cdot 17,0 \cdot 40)$ as well as larger reductions in the intake of the meat-rich diet $(-0 \cdot 12$; $95 \%$ CI: $-0.45,0 \cdot 21)$ than those who had non-FD without dentures. However, these differences were not statistically significant.

\section{Discussion}

This 12-month longitudinal study provided little support for the relationship between FD and changes in DP in a sample of Thai older adults. While our analysis by single food 
Table 2 Comparison of 12-month changes in daily intake (servings/d) of 22 food groups by functional dentition (FD) groups in Thai older adults $(n 651)$

\begin{tabular}{|c|c|c|c|c|c|c|c|c|c|c|c|}
\hline \multirow[b]{2}{*}{ Food groups } & \multicolumn{3}{|c|}{ FD-/D- $(n$ 428) } & \multicolumn{4}{|c|}{$\mathrm{FD}-/ \mathrm{D}+(n$ 131) } & \multicolumn{4}{|c|}{$\mathrm{FD}+(n 92)$} \\
\hline & Mean & SD & Coef. $^{*}$ & Mean & SD & Coef. $^{*}$ & $95 \% \mathrm{Cl}$ & Mean & SD & Coef. ${ }^{*}$ & $95 \% \mathrm{Cl}$ \\
\hline Rice & 0.11 & 1.25 & Reference & -0.02 & $1 \cdot 24$ & 0.03 & $-0.15,0.21$ & -0.01 & $1 \cdot 12$ & -0.07 & $-0.28,0.13$ \\
\hline Noodles & 0.04 & 0.37 & Reference & 0.07 & 0.23 & -0.01 & $-0.04,0.03$ & 0.04 & 0.34 & 0.03 & $-0.02,0.07$ \\
\hline Wheat & 0.08 & 0.25 & Reference & 0.06 & $0 \cdot 18$ & -0.03 & $-0.07,0.02$ & 0.09 & 0.24 & 0.01 & $-0.04,0.06$ \\
\hline Legumes & 0.04 & 0.44 & Reference & 0.00 & 0.34 & -0.01 & $-0.08,0.06$ & -0.01 & 0.41 & -0.03 & $-0.12,0.05$ \\
\hline Vegetables & -0.33 & 1.44 & Reference & -0.27 & 1.23 & 0.03 & $-0.08,0.13$ & -0.29 & 0.89 & $0 \cdot 16$ & $0.04,0.28 \dagger$ \\
\hline Fruits & 0.28 & 0.80 & Reference & 0.36 & 0.71 & 0.04 & $-0.08,0.15$ & 0.30 & 0.75 & 0.07 & $-0.07,0.20$ \\
\hline Red meat & -0.10 & 0.65 & Reference & -0.14 & 0.77 & 0.00 & $-0.07,0.08$ & -0.31 & 0.94 & -0.01 & $-0.10,0.08$ \\
\hline Poultry & -0.01 & 0.38 & Reference & 0.02 & 0.28 & 0.01 & $-0.03,0.05$ & -0.03 & 0.46 & 0.00 & $-0.05,0.05$ \\
\hline Fatty meat & 0.02 & 0.19 & Reference & -0.01 & 0.32 & -0.01 & $-0.03,0.02$ & 0.03 & 0.16 & -0.01 & $-0.04,0.02$ \\
\hline Fried food & -0.01 & 0.15 & Reference & -0.01 & $0 \cdot 10$ & 0.01 & $-0.02,0.04$ & 0.00 & 0.02 & 0.00 & $-0.03,0.03$ \\
\hline Animal organs & 0.00 & 0.06 & Reference & 0.00 & 0.04 & 0.00 & $-0.01,0.00$ & -0.01 & 0.08 & 0.00 & $-0.01,0.01$ \\
\hline Processed meat & -0.02 & 0.14 & Reference & -0.02 & 0.07 & 0.00 & $0.00,0.00$ & -0.01 & 0.06 & 0.00 & $-0.01,0.00$ \\
\hline Fish & -0.02 & 1.07 & Reference & 0.04 & 0.78 & 0.01 & $0.09,0.10$ & 0.09 & 0.69 & 0.01 & $-0 \cdot 10,0 \cdot 12$ \\
\hline Shellfish and squid & 0.00 & 0.34 & Reference & 0.02 & 0.26 & 0.00 & $-0.03,0.04$ & -0.02 & 0.27 & 0.00 & $-0.04,0.05$ \\
\hline Eggs & 0.25 & 0.73 & Reference & 0.24 & $0 \cdot 80$ & -0.04 & $-0.15,0.07$ & 0.18 & 0.91 & 0.01 & $-0.12,0.14$ \\
\hline Milk & -0.08 & 0.56 & Reference & $-0 \cdot 16$ & 0.55 & 0.03 & $-0.06,0.11$ & -0.14 & 0.61 & -0.01 & $-0.10,0.08$ \\
\hline Soymilk & 0.32 & 0.64 & Reference & 0.36 & 0.61 & -0.01 & $-0.12,0.10$ & 0.28 & 0.60 & -0.04 & $-0.17,0.08$ \\
\hline Fermented fish/soybean & -0.02 & 0.30 & Reference & 0.01 & $0 \cdot 10$ & 0.00 & $-0.02,0.02$ & 0.03 & 0.13 & 0.02 & $-0.01,0.04$ \\
\hline Sweetened beverages & -0.02 & 0.72 & Reference & $-0 \cdot 10$ & 0.73 & -0.07 & $-0.17,0.04$ & -0.01 & 0.59 & -0.04 & $-0.17,0.08$ \\
\hline Sweet snacks & 0.05 & 0.85 & Reference & 0.16 & 0.83 & 0.01 & $-0.10,0.12$ & 0.12 & 0.86 & -0.07 & $-0.20,0.06$ \\
\hline Chilli sauce dip & 0.10 & 0.79 & Reference & 0.09 & 0.90 & 0.04 & $-0.06,0.14$ & -0.05 & 0.78 & -0.03 & $-0.14,0.09$ \\
\hline Food with coconut milk & 0.04 & 0.34 & Reference & 0.03 & 0.34 & -0.01 & $-0.05,0.04$ & 0.03 & 0.32 & 0.00 & $-0.05,0.06$ \\
\hline
\end{tabular}

FD-/D-, non-FD without dentures; FD-/D+, non-FD with dentures.

*Linear regression model was fitted for each food group separately.

†Regression coefficients (Coef.) were adjusted for baseline age groups, gender, residence area, education, wealth quartiles, smoking status, physical activity, hypertension, hyperlipidaemia, diabetes, medications, total energy intake and food group intake.

Table 3 Rotated solution from factor analysis of twenty-two food groups among Thai older adults $(n 651)^{*}$

\begin{tabular}{lccc}
\hline Food groups & $\begin{array}{c}\text { Pattern 1: } \\
\text { healthy } \\
\text { diet }\end{array}$ & $\begin{array}{c}\text { Pattern 2: } \\
\text { meat-rich } \\
\text { diet }\end{array}$ & $\begin{array}{c}\text { Pattern 3: } \\
\text { carbohydrate-rich } \\
\text { diet }\end{array}$ \\
\hline Rice & & & -0.682 \\
Noodles & & 0.660 \\
Wheat \\
$\begin{array}{l}\text { Legumes } \\
\text { Vegetables }\end{array}$ & 0.818 & & \\
$\begin{array}{l}\text { Fruits } \\
\text { Red meat }\end{array}$ & 0.375 & 0.473 & 0.349 \\
$\begin{array}{l}\text { Poultry } \\
\text { Fatty meat }\end{array}$ & 0.334 & 0.535 & \\
$\begin{array}{l}\text { Fried food } \\
\text { Animal organs }\end{array}$ & 0.316 & & \\
$\begin{array}{l}\text { Processed meat } \\
\text { Fish }\end{array}$ & 0.421 & & 0.346 \\
$\begin{array}{l}\text { Shellfish and squid } \\
\text { Eggs }\end{array}$ & 0.723 & 0.343 & \\
$\begin{array}{l}\text { Milk } \\
\text { Soymilk }\end{array}$ & & 0.285 & \\
$\begin{array}{l}\text { Fermented fish/ } \\
\text { soybean }\end{array}$ & 0.499 & 0.570 & \\
Sweetened \\
beverages
\end{tabular}

${ }^{*}$ Factor analysis was used to determine the number of patterns to be retained. groups revealed an association between baseline FD and changes in intake of vegetables after adjustment for confounders, our analysis of the whole diet of participants could not replicate such a finding.

The lack of association between FD and most individual food groups was not surprising. A recent systematic review of longitudinal studies found no consistent associations between tooth loss and consumption of food groups across the eight studies included ${ }^{(5)}$. This finding agrees with two previous studies where adults with impaired dentition ( $\leq 5 \mathrm{FTU}$ ) had greater declines in intake of vegetables and those with $<21$ teeth had lower future intake of vegetables ${ }^{(35)}$ than their corresponding counterparts. Taken together, these findings build evidence on the nutritional pathway (from missing teeth to unhealthy diet to chronic conditions) that has been proposed to explain the links between oral health and chronic conditions.

Our whole diet assessment, using factor analysis, identified three mutually exclusive (uncorrelated) DP that characterised participants' diet. These patterns were consistent with those reported in previous analysis of Thai nutrition surveys ${ }^{(27,28)}$. These similarities are noteworthy given the variations in the length of the FFQ (much shorter in previous studies) and the age of participants (middle-aged adults in previous studies) among studies. Unlike younger generations in Thailand who have been more exposed to a Western diet due to globalisation, older adults still rely on traditional diets based on starchy staples and fibre-rich 
Table 4 Regression models for the association between baseline functional dentition (FD) and 12-month changes in dietary pattern scores among Thai older adults $(n 651)^{\star}$

\begin{tabular}{|c|c|c|c|c|c|c|}
\hline \multirow[b]{2}{*}{ FD } & \multirow[b]{2}{*}{ Mean } & \multirow[b]{2}{*}{ SD } & \multicolumn{2}{|c|}{ Crude associations } & \multicolumn{2}{|c|}{ Adjusted associations } \\
\hline & & & Coef. $†$ & $95 \% \mathrm{Cl}$ & Coef. $†$ & $95 \% \mathrm{Cl}$ \\
\hline \multicolumn{7}{|c|}{ Factor 1 : healthy diet } \\
\hline FD-/D- & 0.13 & $1 \cdot 18$ & Reference & & Reference & \\
\hline FD-/D+ & 0.17 & 1.06 & 0.04 & $-0.19,0.27$ & 0.02 & $-0.20,0.25$ \\
\hline $\mathrm{FD}+$ & 0.34 & $1 \cdot 24$ & 0.21 & $-0.06,0.47$ & 0.13 & $-0.13,0.39$ \\
\hline \multicolumn{7}{|c|}{ Factor 2: meat-rich diet } \\
\hline FD-/D- & -0.12 & 1.50 & Reference & & Reference & \\
\hline FD-/D+ & -0.18 & 1.33 & -0.06 & $-0.34,0.23$ & -0.06 & $-0.34,0.22$ \\
\hline FD+ & -0.14 & 1.51 & -0.01 & $-0.34,0.32$ & -0.12 & $-0.45,0.21$ \\
\hline \multicolumn{7}{|c|}{ Factor 3: carbohydrate-rich diet } \\
\hline FD-/D- & 0.62 & $1 \cdot 30$ & Reference & & Reference & \\
\hline FD-/D+ & 0.59 & 1.13 & 0.03 & $-0.22,0.28$ & -0.03 & $-0.28,0.22$ \\
\hline FD+ & 0.72 & 1.29 & 0.13 & $-0.21,0.47$ & 0.12 & $-0.17,0.40$ \\
\hline
\end{tabular}

FD-/D-, non-FD without dentures; FD-/D+, non-FD with dentures.

${ }^{\star}$ The adjusted coefficients were controlled for baseline age groups, gender, residence area, education, wealth quartiles, smoking status, physical activity, hypertension, hyperlipidaemia, diabetes, medications, total energy intake and dietary pattern score.

†Linear regression was fitted for each dietary pattern separately, from which regression coefficients (Coef.) were reported.

foods (especially those living in rural areas). Interestingly, the prudent pattern (i.e. often characterised by high consumption of vegetables, fruits, fish, legumes, whole grains, fish and poultry) was identified in all three Thai studies, suggesting its generalisability and applicability to the local population. Also, while findings from our dietary pattern analysis underline the well-known elements of the Thai cuisine (i.e. a diverse mix of slow-cooked meats with steamed vegetables and rice as the main side dish), they also highlight some of their unhealthy elements such as traditional sweets and desserts (e.g. steamed egg custard and fruits with added sugars) and fried food (e.g. deep fried polished rice and deep fried pork skin).

One explanation for the lack of association between FD and changes in dietary intake could be the relatively short follow-up time (12 months). In that regard, some of the non-significant trends identified in this study could become significant if they are extended (projected) over time. Therefore, the present findings only correspond to shortterm effects of having an FD in later life. In addition, participants did not lose their FD at the baseline assessment, but most have lived without an FD for variable periods of time before the baseline assessment. Indeed, some participants might have experienced change in dentition long time ago and already adapted their food consumption before the study was conducted. Thus, any impact of having a non-FD on diet may have already happened by the time of the baseline assessments and their diet may not have changed, or be expected to change, during the 12-month follow-up period.

The present findings have some implications for practice and research. They support the value of a whole diet assessment over the traditional analysis of specific nutrients or food groups. It provides a comprehensive evaluation of how individuals consume foods, which is especially relevant among Thai families where members gather around a table to enjoy a variety of freshly prepared foods. As for research, the present findings await confirmation from further longitudinal studies, especially those with longer follow-ups and including more waves of dietary data. It would also be an advantage to include multiple dental assessments to capture how changes from an FD to a non-FD or replacing lost teeth with removable complete or partial dentures can impact dietary intake in the long term.

The prospective design, the objective assessment of FD and the use of a validated FFQ for Thai older adults are the strengths of this study. Some limitations are also noted. First, despite the longitudinal design, we are only reporting associations not causal relationships. Second, selection bias could have arisen from two sources, sample selection and attrition. Although the response rate was high, the response at sub-district and province levels were moderate only, which could explain the differences observed with the wider older population. In addition, there was evidence that those lost to follow-up were different than those retained in the sample. These differences imply that the findings cannot be generalised beyond the study sample. Third, even when a validated semi-structured FFQ with a short recall time (previous month) was used for dietary assessment, responses relied on participants' memory, judgements on frequency of intake and portion sizes. Responses could have also been influenced by seasonal variation as some fruits are only consumed in the Summer such as mango, durian, rambutan, longan, custard apple, mangosteen and lychee. In addition, a missing element in the semi-structured FFQ was the cooking method, which could impact on estimation of nutrients due to cooking and increased temperature.

In conclusion, this 12-month longitudinal study among Thai older adults provided little support for an association between baseline FD and changes in DP. Analyses by 
specific food groups showed that having a FD was positively associated with larger increases in intake of vegetables.

\section{Acknowledgements}

Acknowledgements: The authors would like to thank Phetchaburi health authorities and local healthcare professions for supporting this study. Financial support: The follow-up examination was funded by Thailand's Government Expenditure in 2019. Conflict of interest: There are no conflicts of interest. Authorship: Conceptualisation, methodology: P.G., E.B., W.S.; data curation: P.G.; formal analysis, writing original draft preparation: P.G., E.B.; writing - review and editing, supervision: E.B., W.S. Ethics of human subject participation: This study was conducted according to the guidelines laid down in the Declaration of Helsinki, and all procedures involving research study participants were approved by the Biomedical Sciences, Dentistry, Medicine and Natural \& Mathematical Sciences Research Ethics Sub-Committee (BDM RESC) of King's College London (HR-17/18-4631) and Phetchaburi Health Authority (COA No.001/2561). Written informed consent was obtained from all participants.

\section{Supplementary material}

For supplementary material accompanying this paper visit https://doi.org/10.1017/S1368980020004279

\section{References}

1. World Health Organization (2017) Global Strategy and Action Plan on Ageing and Health. Geneva: World Health Organization.

2. Critselis E \& Panagiotakos D (2019) Adherence to the Mediterranean diet and healthy ageing: current evidence, biological pathways, and future directions. Crit Rev Food Sci Nutr 60, 1-10.

3. Meurman JH, McKenna G, Murtomaa H et al. (2018) Managing our older population: the challenges ahead. J Dent Res 97, 1077-1078.

4. Tada A \& Miura H (2014) Systematic review of the association of mastication with food and nutrient intake in the independent elderly. Arch Gerontol Geriatr 59, 497-505.

5. Gaewkhiew P, Sabbah W \& Bernabe E (2017) Does tooth loss affect dietary intake and nutritional status? A systematic review of longitudinal studies. J Dent 67, 1-8.

6. Kiesswetter E, Poggiogalle E, Migliaccio S et al. (2018) Functional determinants of dietary intake in communitydwelling older adults: a DEDIPAC (DEterminants of DIet and Physical ACtivity) systematic literature review. Public Health Nutr 21, 1886-1903.

7. Kayser AF (1981) Shortened dental arches and oral function. J Oral Rehabil 8, 457-462.
8. Witter DJ, van Palenstein Helderman WH, Creugers NH et al. (1999) The shortened dental arch concept and its implications for oral health care. Community Dent Oral Epidemiol 27, 249-258.

9. Iwasaki M, Yoshihara A, Ogawa H et al. (2016) Longitudinal association of dentition status with dietary intake in Japanese adults aged 75 to 80 years. J Oral Rehabil 43, 737-744.

10. Hu FB (2002) Dietary pattern analysis: a new direction in nutritional epidemiology. Curr Opin Lipidol 13, 3-9.

11. Tucker KL (2010) Dietary patterns, approaches, and multicultural perspective. Appl Physiol Nutr Metab 35, 211-218.

12. Burggraf C, Teuber R, Brosig S et al. (2018) Review of a priori dietary quality indices in relation to their construction criteria. Nutr Rev 76, 747-764.

13. Waijers PM, Feskens EJ \& Ocké MC (2007) A critical review of predefined diet quality scores. Br J Nutr 97, 219-231.

14. Newby PK \& Tucker KL (2004) Empirically derived eating patterns using factor or cluster analysis: a review. Nutr Rev 62, 177-203.

15. Asghari G, Mirmiran P, Yuzbashian E et al. (2017) A systematic review of diet quality indices in relation to obesity. $\mathrm{BrJ}$ Nutr 117, 1055-1065.

16. Kant AK (2010) Dietary patterns: biomarkers and chronic disease risk. Appl Physiol Nutr Metab 35, 199-206.

17. Hodkinson HM (1972) Evaluation of a mental test score for assessment of mental impairment in the elderly. Age Ageing 1, 233-238.

18. Katz S, Ford AB, Moskowitz RW et al. (1963) Studies of illness in the aged. The index of ADL: a standardized measure of biological and psychosocial function. JAMA 185, 914-919.

19. Guo Y, Logan HL, Glueck DH et al. (2013) Selecting a sample size for studies with repeated measures. BMC Med Res Methodol 13, 100.

20. Gaewkhiew P, Sabbah W \& Bernabé E (2020) Functional dentition and 12-month changes in body measurements among Thai older adults. Int J Environ Res Public Health 17, 4200

21. Global Adult Tobacco Survey Collaborative Group (2011) Tobacco Questions for Surveys: A Subset of Key Questions from the Global Adult Tobacco Survey (GATS), 2nd ed. Atlanta, GA: Center for Disease Control and Prevention.

22. Banks E, Lim L, Seubsman SA et al. (2011) Relationship of obesity to physical activity, domestic activities, and sedentary behaviours: cross-sectional findings from a national cohort of over 70,000 Thai adults. BMC Public Health 11, 762 .

23. Aekplakorn V, Pakcharoen H, Thaikla K et al. (2014) The 5th Thai National Health Examination Survey 2014. Nonthaburi: Health Systems Research Institute.

24. Banjong O, Chitchaeng U \& Rojroongwasinkul N (2011) Thai elderly Food Frequency Questionnaire: Institution of Nutrition, Mahidol University.

25. Chawaratrungsun A (2015) Validation of Semi-Quantitative Food Frequency Questionnaire to Assess Energy and Macronutrient Intake of Thai Elderly. Masters: Mahidol University.

26. Phromma K (2016) The Validation of a Semi-Quantitative Food Frequency Questionnaire to Assess the Micronutrient Intake of Calcium and Iron Among Elderly Thais. Master: Mahidol University.

27. Aekplakorn W, Satheannoppakao W, Putwatana P et al. (2015) Dietary pattern and metabolic syndrome in Thai adults. J Nutr Metab 2015, 468759.

28. Papier K, Jordan S, D'Este C et al. (2017) Social demography of transitional dietary patterns in Thailand: prospective evidence from the Thai cohort study. Nutrients 9, 1173.

29. WHO (2013) Oral Health Surveys: Basic Methods, 5th ed. Geneva: World Health Organization. 
30. Grace AM \& Smales FC (1989) Mobility and overall destruction. In Periodontal Control: An Effective System for the Diagnosis, Selection and Treatment in General Practice, 1st ed., pp. 52 [AM Grace \& FC Smales, editors]. London: Quintessence Pub. Co.

31. Gaewkhiew P, Sabbah W \& Bernabe E (2019) Functional dentition, dietary intake and nutritional status in Thai older adults. Gerodontology 36, 276-284.

32. Northstone K \& Emmett PM (2008) A comparison of methods to assess changes in dietary patterns from pregnancy to 4 years post-partum obtained using principal components analysis. Br J Nutr 99, 1099-1106.
33. Elstgeest LE, Mishra GD \& Dobson AJ (2012) Transitions in living arrangements are associated with changes in dietary patterns in young women. J Nutr 142, 1561-1567.

34. Togo P, Osler M, Sørensen TI et al. (2004) A longitudinal study of food intake patterns and obesity in adult Danish men and women. Int J Obes Relat Metab Disord 28, 583-593.

35. Logan D, McEvoy CT, McKenna G et al. (2020) Association between oral health status and future dietary intake and diet quality in older men: the PRIME study. J Dent 92, 103265. 